\title{
Experiencia del profesional de enfermería en su relación con el estudiante durante su práctica clínica
}

\section{Experience of the nursing professional in his relationship with the student during clinical practice}

\section{Experiência do profissional de enfermagem em sua relação com o aluno durante a prática clínica}

\author{
Humberto Elizalde-Ordoñez ${ }^{1}$ \\ (iD) https://orcid.org/0000-0002-3157-5603 \\ Gabriela Ortiz-Dávalos ${ }^{2}$ \\ (iD) https://orcid.org/0000-0001-5121-9465 \\ Fanny Rodríguez-Quezada ${ }^{3}$ \\ (iD) https://orcid.org/0000-0001-6573-6543 \\ Kevin Julian Aya-Roa ${ }^{4}$ \\ (D) https://orcid.org/0000-0002-8430-0785 \\ María Mercedes Moreno-González ${ }^{5}$ \\ (iD) https://orcid.org/0000-0002-0299-2299
}

1. Magister en Enfermería Clínico-Quirúrgica. Universidad Técnica Particular de Loja. Loja, Ecuador.

2. Magíster en Educación Especial. Docente Carrera de Enfermería. Universidad Católica. Cuenca, Ecuador.

3. Magíster en Gerencia en Salud para el Desarrollo Local. Universidad Católica. Cuenca, Ecuador.

4. Magister en Ciencias en enfermería. Universidad de Guanajuato. Guanajuato, México.

5. Doctor en Ciencias de Enfermería. Universidad de Guanajuato. Departamento de Enfermería y Obstetricia. Guanajuato, México.

*Autor para correspondencia: kj.ayaroa@ugto.mx

Recibido: 19/03/2020

Aceptado: 28/06/2021 


\title{
Resumen
}

Introducción: La interacción de los estudiantes de enfermería con los enfermeros clínicos en su lugar de práctica influye en su proceso de aprendizaje, generando posibles modelos mentales de atención que muy probablemente serán replicados cuando los estudiantes egresen. Objetivo: Comprender la experiencia del profesional de enfermería en su relación con el estudiante durante su práctica clínica. Metodología: Estudio cualitativo fenomenológico, con muestreo por bola de nieve, hasta llegar a saturación de datos. Colecta de datos mediante entrevista no estructurada a profundidad, las entrevistas fueron grabadas y transcritas literalmente para el análisis. Resultados: Participaron 19 enfermeros (4 hombres y 15 mujeres) y se identificaron dos categorías: estar-con y coexistiendo con los estudiantes. Conclusiones: El profesional de enfermería considera que los estudiantes deben comprender y trabajar de manera colaborativa para aprovechar su experiencia y conocimientos, evitar las criticas destructivas, así como respetar la individualidad. Una buena relación entre estudiantes y enfermeros clínicos podría llevar a un mejor aprovechamiento de la práctica.

Palabras clave: Personal de Enfermería en Hospital; Educación en Enfermería; Enfermería práctica (DeCS).

\begin{abstract}
Introduction: The interaction between the nursing students and the clinical nurses in the practice place influences their learning process, generating possibilities, mental models that will very likely be replicated once the students graduate. Objective: Understand the experience of the nursing professional and their relationship with the student during their medical practice. Methodology: Qualitative phenomenological study using snowball sampling, until reaching data saturation. Data collection throughout non-structured in-depth interviews; the interviews were recorded and literally transcribed for analysis. Results: 19 Nurses participated (4 men and 15 women) which were identified into two categories: currently-around and coexisting with the students. Conclusions: The Nursing professional considered that the students should understand and work in a collaborative manner in order to take advantage of their experience and knowledge, prevent crushing remarks, as well as respect individuality. A Good relationship between the students and clinical nurses could lead to a more beneficial practice.
\end{abstract}

Key words: Health Personnel; Health Education; Nursing, Practical (DeCS).

\begin{abstract}
Abstrato
Introdução: A interação dos estudantes de enfermagem com os enfermeiros assistenciais no local de prática influencia seu processo de aprendizagem, gerando possíveis modelos mentais de cuidado que provavelmente serão reproduzidos quando os estudantes se formarem. Objetivo: Compreender a vivência do profissional de enfermagem na sua relação com o aluno durante a sua prática clínica. Metodologia: Estudo qualitativo fenomenológico, com amostragem intencional, até a saturação dos dados. Coleta de dados por meio de entrevista não estruturada em profundidade, as entrevistas foram gravadas e transcritas literalmente para análise. Resultados: 19 participantes (4 homens e 15 mulheres); foram identificadas duas categorias: "Estar-com" e "coexistiendo" com os alunos. Conclusões: $O$ profissional de enfermagem acredita que o aluno deve compreender e trabalhar de forma colaborativa para aproveitar sua experiência e conhecimento, evitar as críticas destrutivas, além de respeitar a individualidade. Um bom relacionamento entre alunos e enfermeiras clínicas pode levar ao melhor uso da prática.
\end{abstract}

Palavras-chave: Pessoal de Saúde; Educação em Saúde; Enfermagem Prática (DeCS). 


\section{Introducción}

En el desarrollo profesional de enfermería se encuentra inmersa la combinación teoría - práctica, que se alinea a través del desarrollo de actividades de promoción, prevención y recuperación de la salud ejecutadas con la guía del docente, desarrollando habilidades, destrezas y vivencias teóricas que respaldan el aprendizaje de los futuros profesionales ${ }^{(1)}$. En este sentido, la práctica clínica es de suma importancia en el proceso formativo de los futuros enfermeros.

La articulación entre las instituciones de salud y de educación son de gran importancia para la enseñanza de la enfermería, dado que el proceso de enseñanza requiere de profesionales clínicos en el lugar de práctica ${ }^{(2-3)}$. Dentro de las instituciones de salud donde los estudiantes realizan sus prácticas, observan y aprenden del trato existente entre docentes y licenciados en enfermería con los sujetos de cuidado, por lo que se espera que mediante el apoyo de estos los estudiantes desarrollen cualidades profesionales mostrando la manera correcta de ejercer una profesión responsable y científica ante los usuarios de atención, sin embargo, el proceso de enseñanza de la enfermería moderna en algunos casos está centrada en la aplicación de técnicas y elementos procedimentales evaluándose paso a paso ${ }^{(4)}$.

En el proceso de enseñanza-aprendizaje, se involucran tres actores: el estudiante, el docente y el enfermero clínico ${ }^{(3)}$, por lo tanto, el escenario hospitalario donde se gestiona la enseñanza de los estudiantes es el lugar ideal para la aplicación de los elementos epistemológicos que fundamentan la ciencia y el humanismo del cuidado ${ }^{(5)}$, para lo cual el enfermero del servicio podría influir y reforzar los elementos esenciales del arte del cuidado en la enseñanza del nuevo profesional en formación, desarticulándose la práctica ideal con la práctica real ejercida a diario en las instituciones de salud.

La razón de ser de la presente investigación se fundamenta en el conocimiento que se puede generar de la experiencia de los enfermeros en su relación con el estudiante durante su práctica clínica, para que en un futuro se puedan generar estrategias para que el enfermero clínico sea más potencializador en el aprendizaje de los estudiantes de enfermería y de esta forma sacar el mayor provecho a las prácticas. 
Para lo anterior, se procedió a utilizar como herramienta investigativa la fenomenología, la cual podría conceder espacio interpretativo al fenómeno que se estudia, para aplicar el objetivo de comprender la combinación de la experiencia vivida, circunscribiendo el significado que esas realidades tienen para los sujetos involucrados, intentando comprender y no explicar ${ }^{(6-7)}$. Mediante ajuste teórico y temático, se organizan las expectativas y especulaciones en el estudiante y el rol específico que cumplen los enfermeros clínicos; la fase investigativa se apunta al enfermero clínico.

Entender la calidad de la enseñanza en la práctica clínica desde la perspectiva de los estudiantes será muy útil en la prestación de mejores experiencias educativas, para ello es necesario comprender como se dan estas vivencias y qué enseñanza del cuidado deja en los estudiantes ${ }^{(8)}$.

Para este caso se buscó la experiencia de los enfermeros clínicos en la relación con los estudiantes en el campo clínico, es decir, cómo se da el transcurso integral del discernimiento y el nivel de afectividad entre sujetos, comprendiendo la combinación de la experiencia vivida para los sujetos involucrados.

Ante la premisa emergen otros interrogantes: ¿Cómo reacciona el enfermero clínico al recibir estudiantes durante sus horas de turno? ¿Siente el enfermero clínico como imposición, tener estudiantes a su cargo? ¿Cómo es la relación con el estudiante durante el tiempo de práctica?

\section{Objetivo}

Comprender la experiencia del profesional de enfermería en su relación con el estudiante durante su práctica clínica.

\section{Metodología}

Estudio cualitativo, descriptivo de carácter fenomenológico; el fenómeno del estudio es la experiencia de los enfermeros clínicos en su relación con los estudiantes de enfermería en su práctica; la fenomenología como herramienta educativa, permite comprender a profundidad sus funciones como educadores, de modo que se puedan tener en cuenta diferentes situaciones que se encuentran por fuera de la condición escolar, pero que son importantes en el proceso de enseñanza-aprendizaje de los futuros profesionales ${ }^{(9)}$. 
Se definieron como características de los participantes: enfermeros hombres y mujeres trabajadores de una institución de segundo nivel de atención con tres años de experiencia laboral en la misma institución (la institución de salud en los servicios donde hay enfermeros clínicos con mayor experiencia es donde permiten acceder a prácticas).

Se recolectó la información en el inicio y finalización del ciclo académico en los meses de marzo - agosto del 2018. El muestreo se desarrolló mediante la técnica de bola de nieve, buscando enfermeros con práctica clínica exclusiva en un hospital de especialidad, en la región litoral, por tener flujo constante de estudiantes provenientes de varias escuelas de enfermería en Ecuador.

Fueron incluidos 19 participantes, se empleó la entrevista semiestructurada como método para la recolección de la información, estas fueron realizadas de forma individual y grabadas en audio previa autorización de los participantes para su posterior interpretación. Antes de la entrevista, se realizó la orientación sobre los aspectos globales de la investigación, reflexionando la fundamentación fenomenológica de la entrevista, cuya aclaración permitió no inducir respuestas, las entrevistas se realizaron en un espacio académico dentro la institución de salud, en un tiempo brindado por los enfermeros, que generalmente era luego de realizar las actividades prioritarias de su jornada laboral en el cuidado de los pacientes.

La pregunta detonadora para que los participantes desarrollaran sus exposiciones fue: ¿Qué experiencia se desarrolla en usted, cuando en la práctica clínica, debe compartir con estudiantes de enfermería? El análisis de los datos del presente estudio se realizó de forma manual, sacando las categorías de acuerdo con los elementos repetitivos encontrados en las entrevistas, este análisis se realizó en colaboración de investigadores de Ecuador con México.

La siguiente investigación cuenta con la aprobación del comité de bioética con número de registro ADOr001V6.

\section{Resultados}

Se incluyeron 19 participantes, (5 hombres y 14 mujeres); la tabla 1 muestra los datos sociodemográficos más sobresalientes. Del análisis realizado emergieron 2 categorías, las cuales se describen a continuación: 


\section{Categoría 1: El estar-con, en la relación con los estudiantes.}

La relación enfermero - estudiante se caracteriza por tener una perspectiva relacional en entornos complejos, donde existen intercambio de saberes, de auto conocimiento y reflexión del significado de ciertos fenómenos y lo que conllevan sus efectos. Muchos enfermeros clínicos asumen un rol docente en la práctica con los estudiantes sin tener relación alguna con la institución de educación, tal vez por vocación, llegando a desarrollar habilidades para la enseñanza de enfermería cuando se logra establecer una relación de empatía con los estudiantes:

N1: "...asumo el deber adquirido, de compartir con los estudiantes mientras cumplo mi jornada laboral". "al estudiante tú, les compartes la experiencia diaria y ellos lo ponen en práctica...".

N4: "...la oportunidad de estar - con, me permite aprender y enseñar..."

N3: "... aprendemos juntos, porque existe relación cordial...".

Tabla 1. Datos sociodemográficos de los participantes

\begin{tabular}{lllll}
\hline Participante & Edad & Sexo & Experiencia & Estado civil \\
\hline N1 & 34 años & Femenino & 7 años & Soltera \\
N2 & 35 años & Femenino & 9 años & Soltera \\
N3 & 29 años & Femenino & 6 años & Unión libre \\
N4 & 30 años & Femenino & 5 años & Unión libre \\
N5 & 44 años & Femenino & 15 años & Soltero \\
N6 & 46 años & Femenino & 14 años & Soltero \\
N7 & 29 años & Masculino & 3 años & Casado \\
N8 & 41 años & Femenino & 11 años & Soltera \\
N9 & 26 años & Femenino & 4 años & Unión libre \\
N10 & 30 años & Femenino & 6 años & Casada \\
N11 & 28 años & Femenino & 7 años & Casada \\
N12 & 45 años & Femenino & 13 años & Casada \\
N13 & 52 años & Femenino & 20 años & Viuda \\
N14 & 54 años & Masculino & 21 años & Divorciado \\
N15 & 52 años & Femenino & 19 años & Casada \\
N16 & 44 años & Femenino & 10 años & Casada \\
N17 & 33 años & Masculino & 9 años & Soltero \\
N18 & 29 años & Masculino & 5 años & Unión libre \\
N19 & 29 años & Masculino & 13 años & Casado \\
\hline
\end{tabular}

Fuente: Elaboración propia. 
El enfermero y el estudiante en la fase de estar - con, muestran empatía y preocupación para el desarrollo de destrezas en la práctica clínica. Sin embargo, esto no sucede con todo el personal de enfermería que labora en la institución de salud a quienes les asignan estudiantes, dado que en algunas narraciones expresan que algunos no poseen vocación para la enseñanza.

N3: "... mis colegas, que se olvidan su fase de estudiantes...".

N7: "...cuando los alumnos son humillados yo me pongo en sus zapatos..."

N9 "...se debe tener mucho tino para saber cómo tratar a los estudiantes...".

Varios enfermeros expresan que no todos tienen la vocación para impartir docencia, sin embargo, no se excluyen y continúan ayudando a los estudiantes sobre cómo ser y qué hacer en las actividades docente.

N3:" yo hago lo que puedo, no soy experta en docencia, pero me gusta compartir con las nuevas generaciones...recuerdo que en mis tiempos no teníamos tutores, además mi responsabilidad es con el hospital no con la universidad".

N4:"no me gusta, ser docente, pero cuando me solicitan ayuda los estudiantes, les explico".

Se evidencian experiencias positivas y negativas, quienes verbalmente dicen que la actividad docente en áreas de atención a pacientes resulta compleja, por cuanto es una actividad no deseada, tampoco impuesta, pero por gratitud a la formación también recibida, se comprometen.

\section{Categoría 2: Coexistiendo con los estudiantes.}

La coexistencia personifica a cada uno en lo suyo, cuando intervienen lo común del diario vivir, en un mismo tiempo, lugar y espacio. Se establecen canales de comunicación pragmáticos, condicionados a expresiones verbales, escritas o mímicas, en situaciones afines o análogas.

N4: "para mí la coexistencia, existen de manera irracional..., cuando llegan los estudiantes, ya estamos preparados o condicionados que nos causarán conflictos, por eso se expresan la acción dominante". 
N7: "propiciar acciones de atención a los estudiantes, implica que voy a enfrentar a conflictos ..., a veces prefiero que no les asignen actividades, así el acto de coincidir en el mismo espacio sea anulado, no me gusta, a veces ellos creen estar a mi nivel".

N5: "les expreso anticipadamente si desean mantenerse de a buenas conmigo, pregunten todo, así evito conflictos y la coexistencia, mejora siempre".

En las narraciones se manifiesta que la coexistencia existe de forma racional, dado que se tiene establecido mentalmente que cuando llegan los estudiantes, llegan los problemas y por esto existe una relación dominante por parte del profesional de enfermería del servicio hospitalario; sólo de esta forma pueden coexistir algunos enfermeros con los estudiantes.

Por otro lado, en la entrevista revivieron elementos de cuando fueron estudiantes y recuerdan la coexistencia vivida entre ellos y los que en ese entonces eran los enfermeros clínicos, manifestando desagrado y un mal ejemplo para ellos en ese entonces:

N5: "me acordare siempre de la ..., porque demuestra que nunca ha sido estudiante, nos minimiza, se cree la gran cosa, se olvida cuando fue estudiante, parece que nació ya graduada".

N6: "cuando fui interno, me gustaba apegarme a las enfermeras que me daban mayor apertura, y copié sus maneras de guiarme, ahora lo aplico a los jóvenes estudiantes que llegan a mi servicio".

N7: "cuando me acercaba a los docentes, trataba de pedirles permiso para hacer procedimientos de enfermería a veces de complejidad muy alta, varios me permitieron, otros no, y por eso copié esos modelos y antes de permitir a algún estudiante que actué solo, le realizo varias preguntas para asegurarme que conoce la parte teórica para ejecutar en la práctica".

Por ejemplo, no se precisa pasar por alto siempre las faltas del estudiante, encubrirlas o sonreír siempre, si no ofertar sus conocimientos y experiencias cordialmente; por parte del estudiante este debe ser receptor activo para asumir y asimilar la enseñanza impartida que luego aplicaran en su vida profesional, cuidando con calidad, empatía, y 
devolviendo a otros estudiantes lo que recibieron de sus colegas, algunas narraciones concuerdan con la premisa anterior:

N6: "yo copiaba en una libretita, lo bueno y lo malo de cada enfermera, y reflexionaba en mi casa, entonces me proyectaba a futuro, desechando las acciones impropias, claro según mi criterio, escogía lo que quería ser y pues hoy soy un enfermero respetado y querido por todos quienes me conocen".

N6: "jamás he querido ser docente, porque mi vocación es la atención directa al paciente, me siento docente, en mi práctica diaria siempre tengo "mis estudiantes", intercambio ciencia y práctica, ellos también me enseñan".

N7: "para mi criterio, ser docente, es un nivel alto, yo miraba a mis docentes y decía, no seré docente porque mi paciencia es limitada. Entonces yo nací para ser enfermera, esa es mi vocación, morir gustosa, para que otros continúen viviendo".

\section{Discusión}

Con base a los resultados obtenidos podemos inferir que, asumir responsabilidades fuera de las competencias laborales específicas (dado que no tiene ninguna obligación laboral especifica con los estudiantes), significa para los enfermeros clínicos, desprenderse de conjeturas relacionales y comprometerse ampliamente en un proceso dinámico en bien del crecimiento del futuro profesional de enfermería.

En un estudio realizado en Chile ${ }^{(8)}$ y otro en Nueva Granada España ${ }^{(9)}$, sobre el cómo se involucran las enfermeras de áreas clínicas y la relación dinámica del proceso formativo de nuevos profesionales, se evidenció que tienen una predisposición por varios factores, la reacción es reprimirse en tal acción aseverando la carga laboral y al sentir que se pueden crear vínculos de conflictos laborales, porque las enfermeras clínicas no tienen dependencia laboral con las universidades. La responsabilidad implícita del enseñar se sujeta en gran medida a la parte experiencial del conocimiento y la capacidad de trasmitir conocimientos. En ambos estudios se coincide en el reconocimiento inadvertido que la institución donde laboran les impide ampliarse a recibir y adiestrar estudiantes ${ }^{(8,9)}$.

Otro aspecto, evidenciado en la relación "estar-con", es el cansancio físico y mental que se puede generar al trabajar y tener estudiantes, pues las fases de atención directa, administrativa, sumada la actividad con los estudiantes 
puede crear esferas de agotamiento. En áreas clínicas la tensión exige otros desafíos, alterando relaciones interpersonales y pudiendo generar desigualdad de las enfermeras clínicas en el trato con los estudiantes.

Hacerse responsables de estudiantes es una función voluntaria de los enfermeros clínicos, que requiere de habilidades personales (competencias técnicas y emocionales) y de un acompañamiento adecuado por parte de estos, además del servicio y del propio centro hospitalario ${ }^{(11)}$; sumada a las competencias profesionales en el servicio de clínica que implica cuidar la vida, pudiendo surgir situaciones mal interpretadas, por ejemplo, la intención de ignorar a los estudiantes durante su práctica.

Los convenios entre academia y áreas sanitarias, ofrecen oportunidades para los estudiantes y apoyo para las instituciones, sin embargo, la investigación aconseja implementar cursos de actualización y enseñanza en tutoría clínica a las docentes que supervisan la práctica, para implementar estrategias que impacten en el acompañamiento y tutoría personalizada a los estudiantes de enfermería en espacios clínicos e implementar como instrumento de reflexión el diario de prácticas clínicas para que se desarrolle el pensamiento crítico en el estudiante. El diario de prácticas clínicas permite el almacenamiento de lo observado de forma organizada, potenciando el desarrollo de estrategias para jerarquizar lo que es prioritario observar ${ }^{(12)}$.

Fortalecer la función de los tutores o en este caso de los enfermeros clínicos por medio de la educación dirigida al uso de habilidades y experiencias apartándose del modelo puramente repetitivo y carente de reflexión, podría impulsar que los estudiantes logren culminar su preparación académica con éxito.

La comunicación verbal y no verbal es básica en el aprendizaje, fomentar el estudio en grupos colaborativos proporcionándoles la oportunidad de participar en su aprendizaje, asimismo, deberá asumir la responsabilidad de desarrollar e implementar estrategias de aprendizaje de acuerdo con las necesidades de cada tutorado, evaluando así la efectividad de estas ${ }^{(13)}$.

Por otra parte, los enfermeros se preguntan y cuestionan la práctica docente, mirando la labor de los que la ejercen, en el sentido de tomar conciencia, de cuánta capacitación, experiencia y gamma de conocimientos deben poseer. La seguridad del que hacer enfermero en las unidades de prácticas, que realizan de los estudiantes, es el 
meollo de interés, por tanto, ¿será que los recién graduados están en capacidad de ejercer tutorías?, realmente se puede conjeturar, y experimentar nerviosismo, por el poco sentido de la realidad objetiva de la preparación total que debe poseer un docente de nivel universitario.

En las narraciones se manifiesta una coexistencia de forma racional, otorgándole un significado negativo al estudiante como sinónimo de problemas, fomentando una relación dominante por parte del profesional de enfermería del servicio hospitalario; solo de esta forma coexisten algunos enfermeros con los estudiantes, el posicionar al estudiante como el "ser presente" que necesita de una excelente preparación en los lugares de práctica para el cuidado de la salud, requiere de la modificación de dichos significados otorgado a los estudiantes.

Se establece que la relación pedagógica entre el estudiante y el enfermero/a desempeña un papel importante en la formación clínica de este, desarrollando en él, razonamiento y habilidades clínicas, elaboración de significados del cuidado, fortalecimiento de las cualidades relacionales y éticas, y reflexiones sobre el acto de cuidar en las situaciones clínicas ${ }^{(15)}$.

Por otro lado, el impacto de la relación entre el personal de enfermería y los estudiantes se ve reflejada en las experiencias cambiantes expuestas en la entrevista, estableciendo una relación empática en el mundo laboral de experiencias similares, percibiendo e intercambiando las vivencias y sentimientos que abrumaron la formación y que abruman a quienes aún se están formando en la práctica clínica de enfermería. Esto se puede evidenciar de igual forma en una investigación realizada en Perú, que indica que los estudiantes de enfermería expresaron que las enfermeras clínicas, no siempre dejan huellas valederas como modelos a seguir ${ }^{(16)}$.

Los profesionales de enfermería, que, tienen la oportunidad de "estar con", las nuevas generaciones de estudiantes y compartir con ellas sus experiencias clínicas, están conscientes del compromiso y reflexionan refiriéndolo en las entrevistas, si en cada uno de los estudiantes siembran buenos o malos recuerdos como fueron sembrado en ellos buenos y malos recuerdos de cuando fueron estudiantes; a la vez las enfermeras "son" y los estudiantes "están", emitiendo juicios desde las perspectivas individuales, respetando a veces por temor, o enseñando por obligación. 
En un estudio similar realizado en México se evidencia que los estudiantes deben ser atendidos, respetados y ayudados, incondicionalmente sin anticiparse a su desempeño, considerando que gran parte del aprendizaje se genera en el área clínica, sugiriendo asistir en todo momento al estudiante, para permitirle desarrollo y crecimiento en todas las esferas del proceso enseñanza-aprendizaje, adecuando el comportamiento para el desarrollo profesional en ámbitos técnico y científico pero también humano, etiquetando la formación desde las normas del buen vivir, en calidad de ser excelente ciudadano, con resultados hacia aspectos sociales, existenciales y fiables a la condición de ofrecer cuidados calidad-humanos-científicos ${ }^{(17)}$.

Según Zavala Olalde(18) , "el ser humano indivisible, único y responsable de sus acciones, buscando soluciones según propia conveniencia, que nadie modifique o interfiera en sus experiencias". Los programas de enfermería vigentes en el país cumplen las normativas estipuladas por los organismos veedores de la Educación Superior en el Ecuador y buscan las áreas de práctica hospitalaria o comunitaria, que presten facilidades a los estudiantes para cumplir la dinámica integradora del cuidado y la interrelación con el paciente, familiares y otras personas miembros del equipo de salud ${ }^{(19)}$.

\section{Conclusiones}

Desde la experiencia de práctica clínica, el profesional de enfermería considera que los estudiantes deben comprender y trabajar de manera colaborativa para aprovechar de sus conocimientos, evitar las críticas destructivas, acerca del temperamento, comportamiento y carácter, así como respetar la individualidad.

Se resalta la finalización en el sentido del cooperar con enfermeras y estudiantes, para descubrir el origen fenomenológico que repetitivamente llega y se desvanece, a modo de circulo vicioso indistinto.

Los planes de estudio de enfermería que se desarrollan en el país deben analizar la posibilidad de puntualizar contenidos mínimos curriculares para fortalecer el ser-el quehacer y el saber hacer, enfatizando disipar entornos negativos, que permitan seleccionar la factibilidad de aprendizajes significativos mediante el diálogo y la discusión con el propósito de plasmar estilos formativos hacia la meta profesional. 
La experiencia clínica es un proceso muy importante en la formación de enfermeros profesionales, dentro de la cual la enfermera y/o enfermero clínico tienen un papel fundamental, por tal motivo, se debe conocer la experiencia de estos para saber qué profesionales poseen las competencias y la vocación para enseñar, y quiénes pueden ser potencializadores del aprendizaje, cuando no se tenga un docente todo el tiempo.

Los enfermeros clínicos, el docente y el estudiante juegan un papel fundamental para el aprendizaje del futuro profesional, por tal motivo, las relaciones establecidas en este proceso serán muy útil para brindar de herramientas profesionales a los estudiantes.

\section{Conflicto de intereses}

Los autores declaran no tener conflicto de intereses.

\section{Financiamiento}

La presente investigación se realizó con el financiamiento de los investigadores.

\section{Referencias bibliográficas}

1. Corona L, Fonseca M. Aspectos didácticos acerca de las habilidades como contenido de aprendizaje: Una necesidad impostergable. A MediSur [Internet]. 2009 [Consultado febrero de 2020]; 7 (3): 38-43. Disponible en: http://scielo.sld.cu/scielo.php?script=sci_arttext\&pid=S1727-897X2009000300006\&lng=es\&tlng=es.

2. Bousso RS, Merighi MAB, Rolim MA, Riesco MLG, Ângelo M. Estágio curricular em enfermagem: transição de identidade. Rev Esc Enferm USP [Internet]. 2000 [Consultado octubre de 2020]; 34(2):218-25. Disponible em: http://www.ee.usp.br/reeusp/upload/pdf/517.pdf

3. Ito EE, Takahashi RT. Publicações sobre ensino em enfermagem na revista de enfermagem da Escola de Enfermagem da USP. Rev Esc Enferm USP [Internet]. 2005. [Consultado octubre de 2020]; 39(4):409-16. Disponible en: http://www.scielo.br/pdf/reeusp/v39n4/05.pdf

4. Macedo K, De Almeida C, Freitas G, Markert W. Enfermero-docente: aspectos interrelacionados con su formación. Enfermería Global [Internet]. 2010 [Consultado febrero de 2020]; 9 (19): 1-15 Disponible en: https://revistas.um.es/eglobal/article/view/107291/10 1951 DOI: https://doi.org/10.6018/eglobal.9.2.107291

5. Santos MFD, Merighi MAB, Muñoz LA. La enfermera clínica y las relaciones con los estudiantes de enfermería: un estudio fenomenológico. Contexto-Enfermagem [Internet] 2010 [Consultado febrero de 2020]; 19(1), 112-119. Disponible en: https://www.scielo.br/scielo.php?script=sci_arttext\&pid=S0104-07072010000100013 DOI: https://doi.org/10.1590/S0104-07072010000100013 
6. Muñoz LA, Cancino F. Bases epistemológicas de la práctica de enfermería. Rev Chil Cs Méd Biol. 1994; 4(7):31-6.

7. Merighi MAB. Reflexões sobre a docência de enfermagem em uma Universidade Pública. Rev Esc Enferm USP. 1998; 32(1):80-3.

8. García E, García A, Reyes J. Relación maestro alumno y sus implicaciones en el aprendizaje. Ra Ximhai [Internet]. 2014 [Consultado febrero de 2020]; 10(5): 279-290. Disponible en: https://www.redalyc.org/pdf/461/46132134019.pdf

9. Valenzuela S. La práctica de enfermería como foco de reflexión. Aquichan [Internet]. 2016 [Consultado febrero de 2020]; 16(4), 415-417.Disponible en: https://aquichan.unisabana.edu.co/index.php/aquichan/article/view/7175/4470 DOI: 10.5294/aqui.2016.16.4.1

10. Pinzón D. Distancias entre la teoría y la práctica en el desarrollo profesional de enfermería. Unimilitar [Internet]. 2017 [Consultado febrero de 2020]; 1-30. Disponible en: http://hdl.handle.net/10654/17088

11. Bonasa M. Factores de riesgo y factores de protección para garantizar el bienestar psicológico y su calidad de vida laboral. Universidad Rovira i Virgili [Internet]. 2016 [Consultado febrero de 2020]; 90-99. Disponible en: https://www.tdx.cat/bitstream/handle/10803/399566/TESI.pdf?sequence=1\&isAllowed=y

12. Uriarte S, Ponce G, Bernal, M. Vivencias cotidianas en espacios clínicos del estudiante de enfermería. Enf. Universitaria [Internet]. 2016 [Consultado febrero de 2020]; 13 (3): 171-177. Disponible en: http://dx.doi.org/10.1016/j.reu.2016.07.002

13. Hidalgo J, Cárdenas M, Rodríguez S. El tutor clínico. Una mirada de los estudiantes de Licenciatura de Enfermería y Obstetricia. Elsevier [Internet]. 2013 [Consultado febrero de 2020]; 10 (3): Recuperado de: https://www.elsevier.es/esrevista-enfermeria-universitaria-400-articulo-el-tutor-clinico-una-mirada-S1665706313726344 DOI:10.1016/S16657063(13)72634-4

14. Vialart N, Medina I, Gavilondo X. La cultura profesional del docente de enfermería: Preparación ante las tecnologías informáticas. Rev. Cubana de Enf. [Internet]. 2018 [Consultado febrero de 2020]; 34(2) . Disponible en: http://revenfermeria.sld.cu/index.php/enf/article/view/1556/359

15. Rivera L. Saber y experiencia de el /la estudiante de enfermería en sus prácticas de cuidado. Universidad de Barcelona [Internet]. 2013 [Consultado febrero de 2020]; 50-58. Disponible en: https://www.tdx.cat/handle/10803/132808

16. Vera M, Cornejo R, Rivas L, Calizaya J, Zamora O, García J. Significado de los valores profesionales en docentes y estudiantes de enfermería, UNMSM - 2014. Anales de la Facultad de Medicina [Internet]. 2014 .[ Consultado febrero de 2020]; 77(3): 225-229. Disponible en: http://dx.doi.org/10.15381/anales.v77i3.12404

17. Moreno M, Prado E, García D. Percepción de los estudiantes de enfermeria sobre el ambiente de aprendizaje durante sus prácticas Clínicas. Rev. cuid [Internet]. 2013 [ Consultado febrero de 2020]; 4(1):444-449. Disponible en: http://www.scielo.org.co/pdf/cuid/v4n1/v4n1a03.pdf

18. Zavala J. La noción general de persona. El origen, historia del concepto y la noción de persona en grupos indígenas de México. Rev. de Hum: Tec. Monterrey [Internet]. 2010 [Consultado febrero de 2020]; 27 (28): 293-318. Disponible en: https://www.redalyc.org/pdf/384/38421211013.pdf 
19. González R. La relación equipo de salud-paciente-familiar. Rev cubana de SP [Internet]. 2006 [Consultado febrero de 2020]; 32(3) Disponible en: http://scielo.sld.cu/scielo.php?script=sci_arttext\&pid=S0864$34662006000300011 \&$ lng =es\&tlng=es.

Cómo citar este artículo: Elizalde-Ordoñez H, Ortiz-Dávalos G, Rodríguez-Quezada F, Aya-Roa KJ, Moreno-González MM. Experiencia del profesional de enfermería en su relación con el estudiante durante su práctica clínica. SANUS [Internet]. 2021 [citado el dd mm aa]; 6:e192. Disponible en: Link/ DOI 